University of New Hampshire

University of New Hampshire Scholars' Repository

Space Science Center

Institute for the Study of Earth, Oceans, and

Space (EOS)

2000

\title{
MARGIE: A gamma-ray burst ultra-long duration balloon mission
}

\author{
D L. Band \\ Los Alamos National Laboratory \\ J L. Matteson \\ University of California - San Diego \\ M L. Cherry \\ Louisiana State University - Baton Rouge \\ J G. Stacy \\ Louisiana State University - Baton Rouge \\ P P. Altice \\ Louisiana State University - Baton Rouge
}

See next page for additional authors

Follow this and additional works at: https://scholars.unh.edu/ssc

\section{Recommended Citation}

MARGIE: A gamma-ray burst ultra-long duration balloon mission Band, D. and Matteson, J. and Cherry, M. and Stacy, J. G. and Altice, P. and Guzik, T. G. and Kappadath, S. C. and Buckley, J. and Hink, P. and Macri, J. and McConnell, M. and Ryan, J. and O'Neill, T. and Zych, A., AIP Conference Proceedings, 526, 696-700 (2000), DOI:http://dx.doi.org/10.1063/1.1361624

This Conference Proceeding is brought to you for free and open access by the Institute for the Study of Earth, Oceans, and Space (EOS) at University of New Hampshire Scholars' Repository. It has been accepted for inclusion in Space Science Center by an authorized administrator of University of New Hampshire Scholars' Repository. For more information, please contact Scholarly.Communication@unh.edu. 


\section{Authors}

D L. Band, J L. Matteson, M L. Cherry, J G. Stacy, P P. Altice, T G. Guzik, S C. Kappadath, J Buckley, P L. Hink, John R. Macri, Mark L. McConnell, James M. Ryan, Terence J. O'Neill, and Allen Zych 


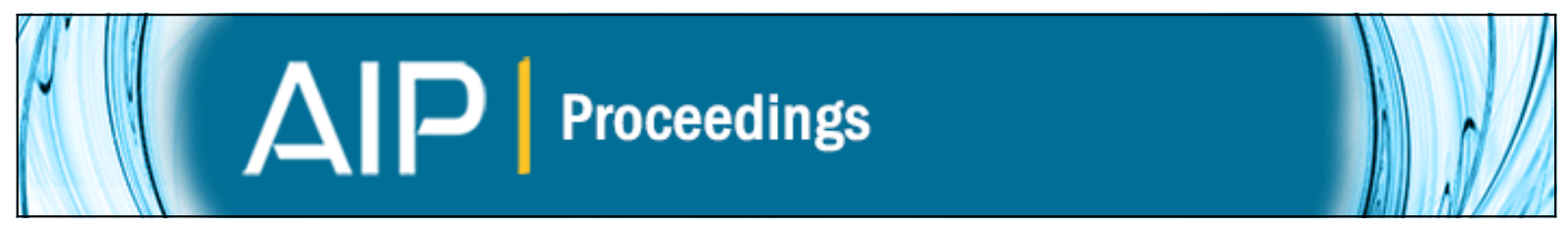

\section{MARGIE: A gamma-ray burst ultra-long duration balloon mission}

D. Band, J. Matteson, M. Cherry, J. G. Stacy, P. Altice, T. G. Guzik, S. C. Kappadath, J. Buckley, P. Hink, J. Macri, M. McConnell, J. Ryan, T. O'Neill, and A. Zych

Citation: AIP Conference Proceedings 526, 696 (2000); doi: 10.1063/1.1361624

View online: http://dx.doi.org/10.1063/1.1361624

View Table of Contents: http://scitation.aip.org/content/aip/proceeding/aipcp/526?ver=pdfcov

Published by the AIP Publishing

\section{Articles you may be interested in}

Response function of the Gamma-Ray Burst Monitor (GRBM) onboard the BeppoSAX satellite

AIP Conf. Proc. 526, 721 (2000); 10.1063/1.1361629

GRB localization with the BeppoSAX Gamma-Ray Burst Monitor

AIP Conf. Proc. 526, 716 (2000); 10.1063/1.1361628

Observations of gamma-ray bursts with MAXI on the international space station

AIP Conf. Proc. 526, 691 (2000); 10.1063/1.1361623

Gamma-ray bursts as a probe of the very high redshift universe

AIP Conf. Proc. 526, 658 (2000); 10.1063/1.1361618

External shock model for gamma-ray bursts during the prompt phase

AIP Conf. Proc. 526, 431 (2000); 10.1063/1.1361576 


\title{
MARGIE: A Gamma-Ray Burst Ultra-Long Duration Balloon Mission
}

\author{
D. Band, ${ }^{1}$ J. Matteson, ${ }^{2}$ M. Cherry, ${ }^{3}$ J. G. Stacy,${ }^{3,4}$ P. Altice, ${ }^{3}$ \\ T. G. Guzik, ${ }^{3}$ S. C. Kappadath ${ }^{3}$ J. Buckley,${ }^{5}$ P. Hink,${ }^{5}$ J. Macri, ${ }^{6}$ \\ M. McConnell, ${ }^{6}$ J. Ryan,${ }^{6}$ T. O'Neill, ${ }^{7}$ and A. Zych ${ }^{7}$ \\ ${ }^{1} X$-2, LANL, Los Alamos, NM 87545 \\ 2 CASS, UC San Diego, La Jolla, CA 92093 \\ ${ }^{3}$ Louisiana State University, Baton Rouge, LA 70803 \\ ${ }^{4}$ Southern University, Baton Rouge, LA 70813 \\ 5 Washington University, St. Louis, MO 63130 \\ ${ }^{6}$ University of New Hampshire, Durham, NH 03824 \\ 7 UC Riverside, Riverside, $C A$ 92521
}

\begin{abstract}
We are designing MARGIE as a 100 day ULDB mission to: a) detect and localize gamma-ray bursts; and b) survey the hard X-ray sky. MARGIE will consist of one small field-of-view (FOV) and four large FOV coded mask modules mounted on a balloon gondola. The burst position will be calculated onboard and disseminated in near-real time, while information about every count will be telemetered to the ground for further analysis. In a 100-day mission we will localize $\sim 40$ bursts with peak photon fluxes from 0.14 to $\sim 5 \mathrm{ph} \mathrm{cm}^{-2} \mathrm{~s}^{-1}$ using $1 \mathrm{~s}$ integrations; the typical localization resolution will be better than $\sim 2$ arcminutes.
\end{abstract}

\section{INTRODUCTION}

An ultra-long duration balloon (ULDB) flight is idcal for a gamma-ray burst mission since the $\sim 100$ day duration is long enough for the detection and localization of a significant burst sample using new technologies which may still be considered too risky for a spacecraft. Consequently we are designing the Minute of Arc Resolution Gamma-ray Imaging Experiment (MARGIE) as a possible ULDB payload. This project is a synthesis of advanced mask construction, detector material and gondola control technologies. Funded by NASA, we are currently in the midst of a 2 year multi-institution design study of the MARGIE concept, and we will propose to build MARGIE when ULDB missions become feasible.

The MARGIE mission will consist of a central imager with a small field-of-view (FOV) and 4 large FOV detectors whose orientations will be offset from that of the central detector. The detectors will be coded mask systems with a detector

CP526, Gamma-Ray Bursts: $5^{\text {th }}$ Huntsville Symposium, edited by R. M. Kippen, et al. (C) 2000 American Institute of Physics 1-56396-947-5/00/\$17.00 
plane of either Cadmium-Zinc-Telluride (CZT) or pixellated CsI scintillators. The detectors will be sensitive over $\sim 50-200 \mathrm{keV}$, with the upper cutoff determined by the detector material and the lower cutoff by the atmosphere. The central detector will have a $43.5 \mathrm{~cm} \times 43.5 \mathrm{~cm}$ detector plane (total area $A_{c e n}=1892.25 \mathrm{~cm}^{2}$ ) with $0.8 \mathrm{~mm} \times 0.8 \mathrm{~mm}$ pixels. Separated from the detector plane by $150 \mathrm{~cm}$, the mask will consist of two cycles of a $521 \times 523$ URA (the mask cells and detector pixels are currently planned to be the same size). The fully-coded FOV will be $16.5^{\circ}$ across and the geometric angular resolution (a mask cell projected on the sky) will be $1.8^{\prime}$. The detector plane of each side-looking detector will be $44 \mathrm{~cm} \times 44 \mathrm{~cm}$ (total area $A_{\text {side }}=1936 \mathrm{~cm}^{2}$ ) with $0.5 \mathrm{~mm} \times 0.5 \mathrm{~mm}$ pixels. The mask will consist of two cycles of a $881 \times 883$ URA (once again, the mask cells and detector pixels will be the same size) separated from the detector plane by $45 \mathrm{~cm}$. The fully-coded FOV will now be $52.1^{\circ}$ across and the geometric angular resolution $3.8^{\prime}$. Surrounding these detectors will be $\sim 10^{5} \mathrm{~cm}^{2}$ of non-imaging $\mathrm{NaI}$ shields which may also be used as burst detectors. An active pointing system is currently not planned, but sub-arcminute aspect information will be provided by fiber optic gyroscopes, CCD star cameras and a GPS receiver. MARGIE will have a total mass of $990 \mathrm{~kg}$ and will consume $760 \mathrm{~W}$ of power.

Previous reports have outlined the details of the proposed instrument and discussed the technologies which will be implemented [1,2]. Here we discuss MARGIE's burst detection and localization capabilities, as well as the possibility of surveying the sky while waiting for a burst to occur.

\section{BURST DETECTION THRESHOLD}

Bursts will be detected by a statistically significant increase (e.g., $\kappa_{b} \sim 5 \sigma$ ) in the count rate accumulated over a time bin of $\Delta t$ (e.g., $1 \mathrm{~s})$. We consider only bursts in the fully-coded FOV.

A number of factors are included in the calculation of the burst count rate. First, the mask blockage is assumed here to be 0.5 . Second, we include the atmospheric attenuation by both photoelectric absorption and Compton scattering; not only the source photons but also the X-ray background photons are attenuated. Finally, we model the detector efficiency.

The background consists of a number of components. First, UCSD's experience with CZT leads us to approximate the internal background as $10^{-4} \mathrm{ct} \mathrm{s}^{-1} \mathrm{~cm}^{-2}$ $\mathrm{keV}^{-1}$ across the energy band of interest. This number will be refined as the project progresses. Second, we use the Gruber parameterization of the diffuse X-ray background [3], attenuated by both the mask and the atmosphere. Third, we include the hard X-rays originating in the atmosphere which consist of both scattered X-ray background photons and cosmic-ray induced emission. We use the flux measured by a UCSD balloon at $3 \mathrm{~g} \mathrm{~cm}^{-2}$ overburden [4]; this is a direct measurement of the atmospheric flux which should not be attenuated for atmospheric absorption.

The calculation of the diffuse background components requires the average solid 
angle $\Omega_{\text {mask }}$ viewed by the detector plane. Using relevant formulae [5] we find $\Omega_{c e n}=0.296 \mathrm{sr}$ for the central detector and $\Omega_{\text {side }}=1.594 \mathrm{sr}$ for each side detector. Note that the atmospheric attenuation, detector efficiency and mask opacity are all energy dependent.

Translated into the BATSE $50-300 \mathrm{keV}$ trigger band using a typical burst spectrum, the threshold peak flux for the central detector should be $\psi_{\text {cen }}=$ $0.14 \Delta t^{-1 / 2}\left(\kappa_{b} / 5 \sigma\right) \mathrm{ph} \mathrm{s}^{-1} \mathrm{~cm}^{-2}$ and for the side detectors $\psi_{\text {side }}=0.30 \Delta t^{-1 / 2}\left(\kappa_{b} / 5 \sigma\right)$ $\mathrm{ph} \mathrm{s}^{-1} \mathrm{~cm}^{-2}$. Using 1) the burst rate, 2) the FOV, 3) a cumulative burst distribution $N(>\psi) \sim \psi^{-0.8}$ at threshold and 4) a mission duration of $M \sim 100$ days, we find that MARGIE should detect $N_{c e n}\left(>\psi_{c e n}\right)=1.5 \Delta t^{0.4}\left(\kappa_{b} / 5 \sigma\right)^{-0.8}(M / 100 \mathrm{~d})$ bursts in the fully coded FOV of the central detector and $N_{\text {side }}\left(>\psi_{\text {side }}\right)=$ $8.6 \Delta t^{0.4}\left(\kappa_{b} / 5 \sigma\right)^{-0.8}(M / 100 \mathrm{~d})$ bursts in each of the side detectors. Accounting for overlapping FOVs, $\sim 25$ bursts should occur in some detector's fully coded FOV. Because the detectors will also detect bursts in the partially coded FOVs, we should detect at least 40 bursts. We assume that each detector triggers independently.

For the fully-coded FOVs, the brightest burst expected in 100 days (i.e., $\psi_{b}$ such that $N\left(>\psi_{b}\right) \sim 1$ ) will have a peak flux of $\psi_{b, c e n}=0.25 \mathrm{ph} \mathrm{cm}^{-2} \mathrm{~s}^{-1}$ and $\psi_{b, s i d e}=4.36 \mathrm{ph} \mathrm{cm}^{-2} \mathrm{~s}^{-1}$ in the central and each of the side detectors, respectively. Of course, with the much larger area covered by all 4 side detectors, including the partially coded regions, the "burst of the mission" will be somewhat larger.

As an aside, note that the all sky burst rate is less than the often quoted 800 bursts $\mathrm{yr}^{-1}$. BATSE's detection threshold is a peak flux of $\psi_{B} \sim 0.3 \mathrm{ph} \mathrm{s}^{-1} \mathrm{~cm}^{-2}$ in the $50-300 \mathrm{keV}$ energy band accumulated on the $1.024 \mathrm{~s}$ timescale. Above $\psi_{B} 772$ bursts were detected in the 3B catalog for which the latest BATSE team live-time is $5.16 \times 10^{7} \mathrm{~s}$ (C. Meegan 1998, personal communication), resulting in $n\left(>\psi_{B}\right)=470 \mathrm{yr}^{-1}$ all sky. Two effects diminish the burst rate: the greater live-time than initially calculated and the threshold of $\psi_{B}=0.3 \mathrm{ph} \mathrm{cm}^{-2} \mathrm{~s}^{-1}$ on the $1.024 \mathrm{~s}$ timescale.

\section{BURST LOCALIZATION}

The ability to localize bursts and the techniques to perform this localization depend on the burst and background count numbers. For these calculations we use bursts with durations of $T_{d}=10 \mathrm{~s}$ and average fluxes $\xi=1 / 3$ of the peak flux. At the detection threshold there should be 190 and 400 burst counts in the central and side detectors, respectively, while for the brightest expected burst 250 and 4525 burst counts are expected in the central and side detectors, respectively. During the burst 1275 central detector and 5745 side detector background counts should also be accumulated. With this small number of counts distributed over a much larger number of pixels $\left(\sim 8 \times 10^{5}\right.$ for the side detectors), back-projection will be the most efficient image reconstruction technique.

There are two localization issues: a) will the burst counts back-project to a statistically significant cluster on the sky; and b) how well can the burst be localized. 
To determine the significance of the cluster of counts back-projected onto the burst's position, consider a grid of possible source positions superimposed on the sky. The pixels of this grid will be the mask cells projected onto the sky. For a mask which is half-open, half-closed, a given count on the detector plane can be back-projected through the mask onto half the grid's pixels. Thus the average count number projected into a non-burst sky pixel will be $\left(n_{G R B}+n_{\text {back }}\right) / 2$, and the standard deviation of this count number will be $\left(n_{G R B}+n_{b a c k}\right)^{1 / 2} / 2$ (the total number of counts is known and therefore the counts per pixel follows the binomial distribution, and not the Poisson distribution; this changes the standard deviation). The expected number of counts in the burst pixel will be $n_{G R B}+n_{\text {back }} / 2$. Thus, the expected difference between the number of counts in the burst and non-burst pixels should be $n_{G R B} / 2$. Consequently, for a burst at threshold the expected sensitivity of a source detection will be $5.0 \sigma$ for the central detector and $5.1 \sigma$ for a side detcctor, while for the brightest expected burst the sensitivities are $6.4 \sigma$ and $44.7 \sigma$ for the central and side detectors, respectively. This sensitivity gives the probability that a fluctuation in a non-source pixel will equal or exceed the counts in the source pixel. Since only positive fluctuations would be mistaken for a source, we use the positive tail of the Gaussian distribution. The probability per pixel is less than $5 \times 10^{-7}$ at threshold for both detectors, but less than $10^{-13}$ for the brightest expected burst for both detector types. However, the number of pixels on the sky is $\sim 3 \times 10^{5}$ for the central detector and $\sim 8 \times 10^{5}$ for each side detector, and therefore there is a probability of $\sim 0.13$ of a source-like fluctuation somewhere in the FOV at threshold, but an infinitesimal probability for the brightest bursts.

Persistent astrophysical sources are probably not an issue. Bursts at threshold will be $0.40 \Delta \iota^{-1 / 2}\left(\kappa_{b} / 5 \sigma\right)\left(\xi / \frac{1}{3}\right)$ Crab for the central detector and 0.83 $\Delta t^{-1 / 2}\left(\kappa_{b} / 5 \sigma\right)\left(\xi / \frac{1}{3}\right)$ Crab for the side detectors. Nonetheless, we will identify steady sources in our FOV to avoid misidentifying bursts.

The rule-of-thumb is that a source can be localized to within the point-spreadfunction divided by the square-root of the source significance. Our point-spreadfunction is the size of a mask cell projected on the sky: $1.8^{\prime}$ for the central detector and $3.8^{\prime}$ for the side detectors. Thus the side detectors should localize threshold bursts to better than $\sim 1.7^{\prime}$.

\section{SURVEY CAPABILITIES}

Between bursts MARGIE will survey the sky. However, the balloon gondola will be an unstable platform. The gondola will swing with a period of $\sim 17.5 \mathrm{~s}$ and a likely amplitude of $\sim 0.5^{\circ}$. In addition, a source will move relative to the earth at $15^{\circ} \cos |\lambda| \mathrm{hr}^{-1}(\lambda$ is the source's declination $)$, or $2.5^{\prime} \cos |\lambda|$ over $10 \mathrm{~s}$, a typical burst duration. However, our aspect determination system should provide sub-arcminute resolution positions.

A given field will be visible for only a fraction of a day but observations from a number of days may be stacked. Techniques exist for compensating for shifts in 
the FOV, although systematic effects may be introduced.

Using the methodology developed for bursts, we find MARGIE's sensitivity to sources is $4.46\left(\kappa_{s} / 5 \sigma\right)\left(T_{s} / 1 \text { day }\right)^{-1 / 2}$ mCrab for the central detector and $9.18\left(\kappa_{s} / 5 \sigma\right)\left(T_{s} / 1 \text { day }\right)^{-1 / 2}$ mCrab for a side detector. At the detection threshold the number of source counts will typically be $5 \times 10^{-2}\left(\kappa_{s} / 5 \sigma\right)\left(T_{s} / 1 \text { day }\right)^{1 / 2}$ counts/detector pixel on top of $50\left(T_{s} / 1\right.$ day) background counts/detector pixel. The image reconstruction will be computationally expensive given the large number of counts accumulated while the FOV shifts by at least the source resolution on short timescales.

The expected number of survey sources is uncertain because the hard X-ray sky has not yet been well surveyed, and extrapolations from less sensitive, lower energy surveys depend on the assumed source spectrum. Galactic sources usually have spectra steeper than $\psi \propto E^{-2}$, but type II AGN may be heavily absorbed at lower energies, and thus will be more numerous at higher energies. In addition, we can point MARGIE at regions which are likely to have a higher hard X-ray density, such as the Galactic Center or the Magellanic Clouds. Nonetheless, it is worth attempting an extrapolation.

We assume a cumulative source distribution of $n(>\psi) \propto \psi^{-\gamma}$, where $\gamma=1$ for Galactic and 3/2 for extragalactic sources. We normalize these distributions using the HEAO-1/A4 catalog [6] -65 Galactic and 8 extragalactic sources in an all-sky survey to a flux limit of $13 \mathrm{mCrab}$ above $13 \mathrm{keV}$ - which is consistent with the HELLAS (Beppo-SAX) 5-10 keV survey [7] for extragalactic sources. We extrapolate these fluxes to higher energies with a Crab spectrum.

The mission duration of $M$ days is broken into $N_{\mathrm{FOV}}$ pointings of $T_{s}$ days each. Clearly, with enough pointings, the whole sky will be covered, which limits $T_{s}>$ $0.66 \mathrm{~d}$ for the central detector and $T_{s}>6.6 \mathrm{~d}$ for each side detector. Thus we expect in 100 days $39.5\left(T_{s} / 10 \mathrm{~d}\right)^{-1 / 2}$ Galactic and $14.8\left(T_{s} / 10 \mathrm{~d}\right)^{-1 / 4}$ extragalactic sources for the central detector and $192\left(T_{s} / 10 \mathrm{~d}\right)^{-1 / 2}$ Galactic and $50\left(T_{s} / 10 \mathrm{~d}\right)^{-1 / 4}$ extragalactic sources for each side detector.

Acknowledgment: This study is supported by NASA grant NAG5-5208.

\section{REFERENCES}

1. Stacy, J. G., et al., in Proc. 19th Texas Symp. on Relativistic Astrophysics, Paris, in press (1999).

2. Cherry, M. L., et al., in Proc. SPIE, submitted (1999).

3. Gruber, D. E., Matteson, J. L., Peterson, L.E., and Jung, G. V., Ap. J. 520, 124 (1999).

4. Matteson, J. L., et al., Sp. Sci. Irnsl. 3, 491 (1977).

5. Sullivan, J. D., Nucl. Inst. Meth. 95, 5 (1971).

6. Levine, A., et al., Ap. J. Suppl. 54, 581 (1984).

7. Fiore, F., ct al., in First XMM Workshop, in press (1999). 